\section{Laparoscopic cholecystectomy without visible scar. Combined suprapubic and transumbilical approach: the "Minden cholecystectomy"}

Natural orifice transluminal endoscopic surgery (NOTES) is a promising technique especially for cholecystectomy. During the last 2 years the combined transvaginal and transumbilical approach without visible scars [1] became a real alternative to standard four-trocar cholecystectomy in several institutions [2-4]. However, clearly this method cannot be used in men, and there might be psychological or cultural barriers preventing women from agreeing to cholecystectomy through the vagina. Therefore, we develinvasive cholecystectomy by transposing the vaginal approach to a suprapubic position in a region normally covered by pubic hair. oped the Minden method for minimally

In November and December 2008, two women (56 and 49 years old, respectively) underwent operations for symptomatic cholecystolithiasis. A $30^{\circ}$ conventional optic (Karl Storz GmbH, Tuttlingen, Germany) was inserted through a $5-\mathrm{mm}$ intraumbilical trocar. A $10-\mathrm{mm}$ trocar was inserted via a suprapubic incision. The gallbladder was fixed to the abdominal wall by a suture ( $\bullet$ Fig. 1 ). The infundibulum was manipulated by a 5 -mm grasper introduced through the suprapubic access alongside the camera trocar in one patient and by a 2 -mm grasper in the second patient. Following dissection of the Calot's triangle via the umbilical access, the cystic duct and cystic artery were clipped with a 5-mm multifire clip device (Ligamax 5 Endoclip applier, Ethicon

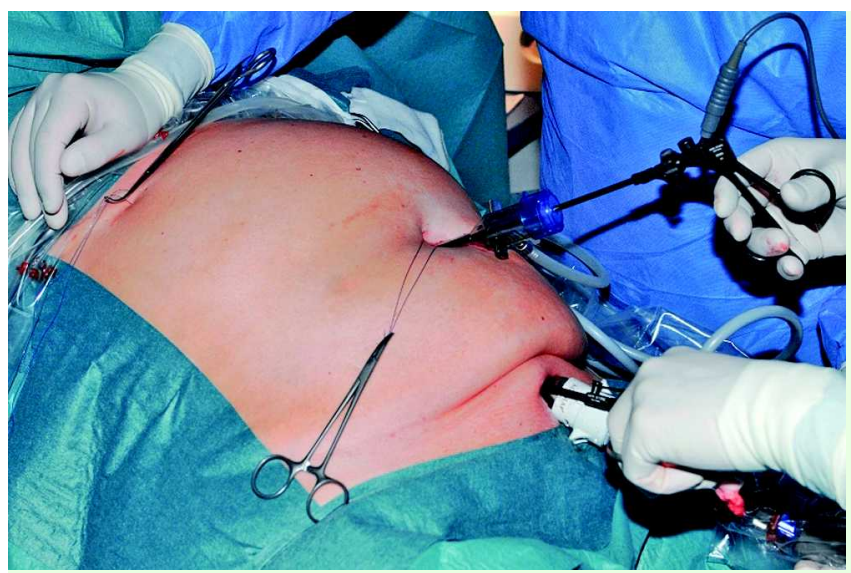

Fig. 1 The operating setting. The dissection instrument is introduced through an umbilical 5-mm trocar. A $30^{\circ}$ camera and a grasper are introduced via a suprapubic incision, and the fundus of the gallbladder is secured to the anterior abdominal wall. The surgeon is standing between the legs of the patient.

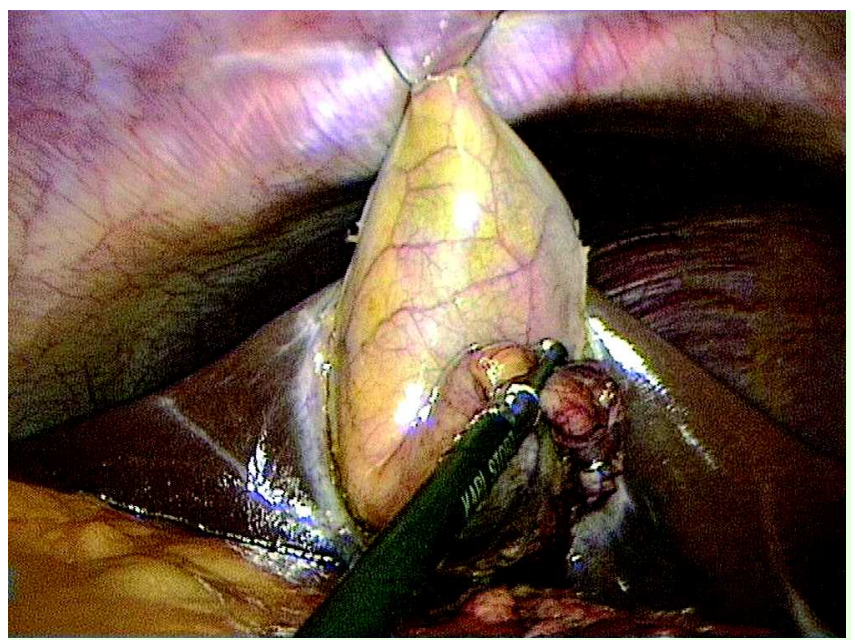

Fig. 2 Intraoperative situs. The cystic artery and the cystic duct are clipped and transected. The fundus of the gallthe anterior abdomina wall by a suture. The infundibulum is manipulated by a grasper, inserted through a suprapubic incision.
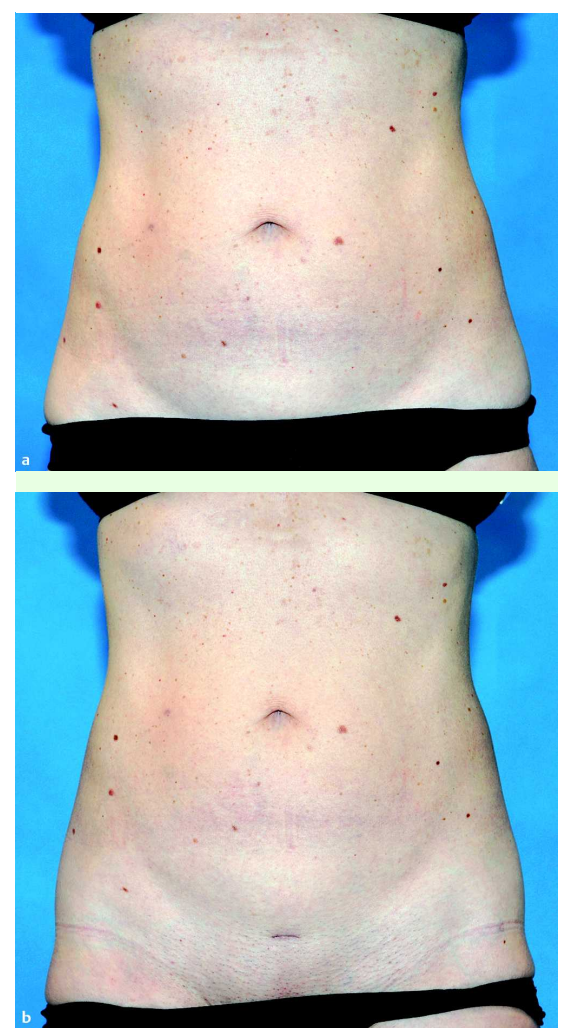

Fig. 3 Postoperative photography of the abdominal wall of the first patient 4 weeks after surgery with (a) and without (b) covering pants: the 5-mm intraumbilical incision is hidden in the umbilicus and the 10-mm suprapubic incision is located in the area of suprapubic hair. The 2-mm puncture for a subsidiary instrument at the right abdominal wall can be seen.

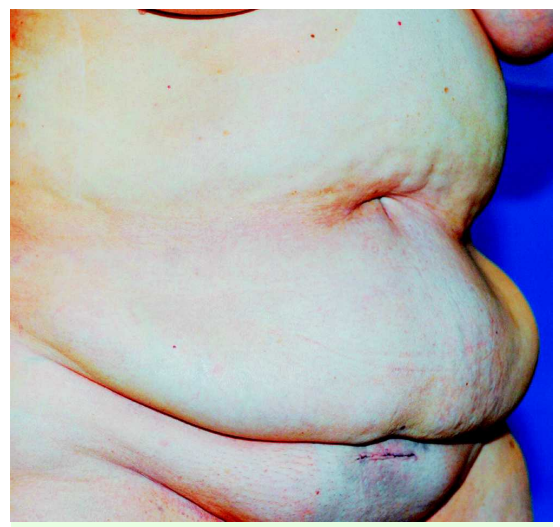

Fig. 4 Postoperative photography of the abdominal wall of the second patient a day after surgery: the 5 -mm intraumbilical incision is hidden in the umbilicus and a 10-mm suprapubic incision is located in the area of suprapubic hair. 
Endo-Surgery Inc., Cincinnati, Ohio, USA) ( Fig. 2). When dissection of the gallbladder was complete it was retrieved by a bag (EMP 100, IFM Gerbershagen, Mindelheim, Germany), which had been inserted through the suprapubic trocar. The operation times were 69 and 89 minutes, respectively, using standard laparoscopic instruments. The postoperative course was uneventful ( $\bullet$ Fig. 3 and 4 ).

Methodologically, the Minden method is only slightly different from conventional laparoscopic cholecystectomy. Thus, in contrast to NOTES techniques using flexible instruments, one can expect a minimal learning curve when trained laparoscopic surgeons switch to the Minden technique, which uses the same instruments as those with which they were trained.

Endoscopy_UCTN_Code_TTT_1AT_2AB
B. Gerdes, E. Gitei, O. Akkermann, J. Prasse-Badde, C. Schmidt

Department of General Surgery, Johannes

Wesling Klinikum Minden, Minden,

Germany

\section{References}

1 Zornig C, Emmermann A, van Waldenfels HA, Mofid H. Laparoscopic cholecystectomy without visible scar: combined transvaginal and transumbilical approach. Endoscopy 2007; 39: 913 - 915

2 Zornig C, Mofid H, Emmermann A et al. Scarless cholecystectomy with combined transvaginal and transumbilical approach in a series of 20 patients. Surg Endosc 2008; 22: $1427-1429$

3 Cardoso RA, Murakami A, Galvao NM et al. NOTES transvaginal video-assisted cholecystectomy: first series. Endoscopy 2008; 40: $572-575$

4 Zornig C, Mofid H, Emmermann A et al. Combined transvaginal and transumbilical approach for cholecystectomy with no visible scarring. Chirurg 2008 Nov 22; Epub ahead of print
Bibliography

DOI $10.1055 / \mathrm{s}-0029-1214483$

Endoscopy 2009; 41: E49-E50

(c) Georg Thieme Verlag KG Stuttgart · New York . ISSN 0013-726X

Corresponding author

B. Gerdes, MD

Johannes Wesling Klinikum Minden Hans Nolte Strasse 1

32429 Minden

Germany

Fax: +49-571-5092716

berthold.gerdes@klinikum-minden.de 